\title{
Interpretation of similarity in the negative polarization of comets and C-type asteroids in terms of common properties of asteroidal and cometary dust
}

\author{
Evgenij Zubko ${ }^{1,2}$ \\ ${ }^{1}$ Department of Physics, P.O. Box 64, FIN-00014, University of Helsinki, Finland \\ ${ }^{2}$ Astronomical Institute of Kharkov National University, 35 Sumskaya St., Kharkov, 61022, Ukraine
}

(Received November 16, 2010; Revised February 28, 2011; Accepted March 14, 2011; Online published February 2, 2012)

\begin{abstract}
At small phase angles, the whole coma aperture-averaged linear polarization of comets nearly coincides with that of C-type asteroids. However, the experimental study of light scattering by independent dust particles and regoliths, consisting of exactly the same particles, shows that their negative polarization branches are substantially different. Therefore, the similarity in the linear polarization of comets and C-type asteroids has to be interpreted in terms of a random coincidence rather than evidence for common properties of dust particles. Imaging polarimetry of comets shows two features with a distinctive behavior of the linear polarization: jets and circumnucleus haloes. Dust particles in jets produce only positive polarization through all the phase angles; whereas, dust particles in the halo reveal a significant negative polarization branch with $P_{\min }=-6 \%$. By comparison with the experimental study of light scattering, such a difference in the negative polarization could indicate common properties of dust in the circumnucleus haloes and C-type asteroids. The high negative polarization can be confidently attributed to weakly absorbing particles. The real part of the refractive index $\operatorname{Re}(m)$ is $1.5-1.6$, and the imaginary part is limited to $\operatorname{Im}(m) \leq 0.02$. The morphology of dust particles in the circumnucleus haloes can be rather fluffy with a material density of about $0.8 \mathrm{~g} / \mathrm{cm}^{3}$. The power-law index for the size distribution is estimated to be about $a=1.5-2$.
\end{abstract}

Key words: Comets, asteroids, negative polarization, discrete dipole approximation.

\section{Introduction}

The most common polarimetry of comets uses measurements of the degree of linear polarization averaged over the whole coma. Although it obviously leads to a loss of spatial information, aperture-averaged polarimetry is much simpler to perform than imaging polarimetry and can be carried out by observatories with relatively-small telescopes and without access to an expensive highly-sensitive charge-coupled device (CCD). For example, the linear polarizations for 13 comets reported by Chernova et al. (1993) were obtained using a 1-m telescope.

One principal result of the whole coma aperture-averaged polarimetry of comets concerns the negative polarization branch (NPB) at small phase angles $\alpha\left(\alpha \leq 20-22^{\circ}\right)$. Note that, in the case of randomly-oriented scatterers, the degree of linear polarization is given by: $P=\left(I_{\perp}-I_{\|}\right) /\left(I_{\perp}+I_{\|}\right)$ (where, $I_{\perp}$ and $I_{\|}$are the intensities of electromagnetic radiation vibrating perpendicular to, and in, the scattering plane respectively). Thus, negative polarization simply implies $I_{\|}>I_{\perp}$ near the backscattering regime. The average shape of the NPB is quite symmetric, so the minimum of the negative polarization $P_{\min }$, typically $\approx-1.7 \%$, is observed at $\alpha_{\min } \approx 10-11^{\circ}$ (e.g., Chernova et al., 1993; Levasseur-

Copyright (c) The Society of Geomagnetism and Earth, Planetary and Space Sciences (SGEPSS); The Seismological Society of Japan; The Volcanological Society of Japan; The Geodetic Society of Japan; The Japanese Society for Planetary Sciences; TERRAPUB.

doi:10.5047/eps.2011.03.005
Regourd et al., 1996). It is important to emphasize that all aperture-averaged polarimetric observations of cometary comae show a NPB at small phase angles; to our knowledge, there are no publications in peer-review journals, reporting an absence of the NPB.

At small phase angles, a synthetic phase curve of the linear polarization of various comets nearly coincides with that found for C-type asteroids. For instance, Fig. 1 presents typical synthetic dependences of the degree of linear polarization $P$ on the phase angle $\alpha$ near backscattering for 10 various comets and $13 \mathrm{C}$-type asteroids. The observations of the comets have been carried out in the continuum part of the spectrum at $\lambda=0.4845-0.53 \mu \mathrm{m}$, except for the emission line of $\mathrm{C}_{2}$ gas around $0.514 \mu \mathrm{m}$. The data for comets are adapted from Chernova et al. (1993), Ganesh et al. (1998), Joshi et al. (2010), Hadamcik and Levasseur-Regourd (2003a), Kikuchi et al. (1987), Kiselev and Chernova (1981), and Rosenbush et al. (1994, 2009a). Most polarimetric data for asteroids have been obtained at $\lambda=0.518 \mu \mathrm{m}$, adapted from Zellner and Gradie (1976).

The resemblance between the NPB of the minor planets and comets was first stressed by Kiselev and Chernova (1981) and later confirmed in, e.g., Chernova et al. (1993) and Levasseur-Regourd et al. (1996). This finding was interpreted in terms of the similar properties of cometary grains and particles forming the regolith in C-type asteroids (e.g., Chernova et al., 1993), which also seems to be consistent with the similar morphology of interplanetary 


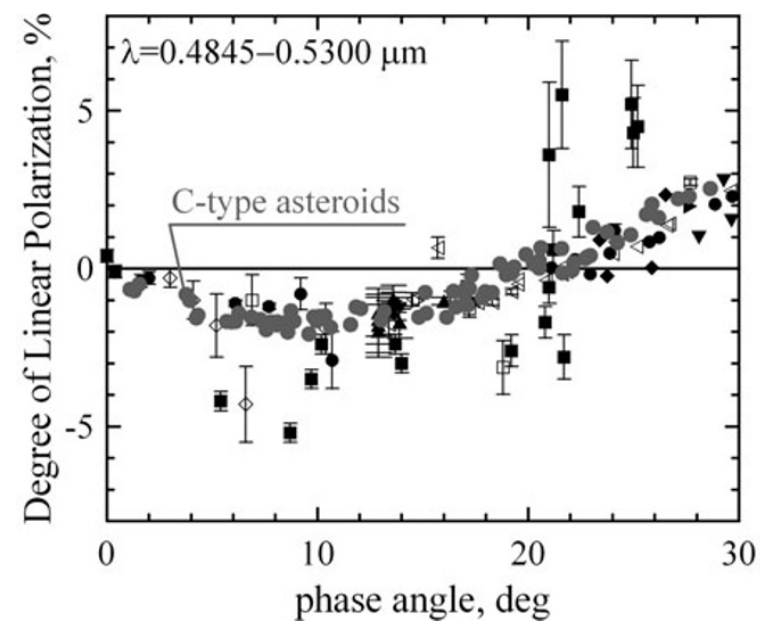

- 1P/Halley

- 4P/Faye

- 17P/Holmes

> $22 \mathrm{P} / \mathrm{Kopff}$

- 47P/Ashbrook-Jackson

- 67P/Churyumov-Gerasimenko

C/1978 H1 (Meier)

$\mathrm{C} / 1989$ XI (Austin)

C/1990 K1 (Levy)

C/1995 O1 (Hale-Bopp)

Fig. 1. Synthetic dependences of the degree of linear polarization on phase angle for comets and C-type asteroids. The data for comets are adapted from Chernova et al. (1993), Ganesh et al. (1998), Joshi et al. (2010), Hadamcik and Levasseur-Regourd (2003a), Kikuchi et al. (1987), Kiselev and Chernova (1981), Rosenbush et al. (1994, 2009a); whereas, data for C-type asteroids are from Zellner and Gradie (1976).

dust particles originating from asteroids and comets (e.g., Brownlee, 1985). Later, the conclusion of a common morphology for cometary and asteroidal dust particles led to the conclusion of the same driving mechanism for the negative polarization found in asteroids and comets (e.g., Petrova et al., 2000; Joshi et al., 2003).

Though the resemblance of asteroidal and cometary IDPs is clear, it is also quite obvious that IDPs collected in the Earth's stratosphere are representative neither of dust in a cometary comae nor regolith particles in C-type asteroids. The coincidence of the negative polarization in asteroids and comets must imply an insensitivity of the negative polarization branch to the physical properties of the target. However, such a "finding" does contradict numerous theoretical studies of light scattering by irregularlyshaped particles, which show that the angular profile of the degree of linear polarization is strongly dependent on the particle properties (e.g., Lumme and Rahola, 1994; Yanamandra-Fisher and Hanner, 1999; Petrova et al., 2000; Kimura, 2001; Kimura et al., 2003; Zubko et al., 2003, 2004, 2005, 2006, 2007, 2008, 2009; Lasue and LevasseurRegourd, 2006; Vilaplana et al., 2006; Muinonen et al., 2007; Lindqvist et al., 2009; Nousiainen, 2009). Laboratory measurements also confirm a strong dependence of the NPB on the properties of dust particles (e.g., Gustafson and Kolokolova, 1999; Muñoz et al., 2000, 2006; Volten et al., 2001; Hadamcik et al., 2009; Renard et al., 2010). Thus, there is a contradiction between the similarities of the NPBs of comets and asteroids, and the high sensitivity of the negative polarization to properties of dust particles.

\section{Negative Polarization Produced by Single- scattering and by Scattering from Dust De- posited on a Surface}

An obvious distinction between the coma and regolith is the number density of particles. The fluffiest regolith remains a substantially more compact medium than the densest cometary coma. In terms of light scattering, it implies a difference in the contribution from multiple scattering. Indeed, in the case of regoliths, the multiple scattering be- tween constituent particles plays an important role and has to be taken into account; whereas, in the case of the coma, it can be simply ignored. Note that a rigorous computation of the multiple scattering in a dense medium consisting of micron-sized particles is a difficult problem. However, the contribution of multiple scattering could be derived from the comparative experimental study of light scattering by independent dust particles and regoliths made of those particles. Shkuratov et al. (2004) present the results of such a study of ten samples of terrestrial dust. Within that work, the same sample particles have been investigated with the nephelometer of the University of Amsterdam (The Netherlands) and the photometer/polarimeter of Kharkov National University (Ukraine). In the former case, scattering from individual sample particles has been studied; whereas, in the latter case, the same particles have been deposited on a surface. By a comparison of the two results, the true contribution of multiple scattering within regoliths can be estimated.

Figure 2 presents the phase curves of the degree of linear polarization for Lokon volcano ash measured in blue light (adapted from Shkuratov et al. (2004)). An SEM image of Lokon volcano ash particles is given in the right panel of Fig. 2 (adapted from Shkuratov et al. (2006)). The deposited sample is characterized by a fluffy structure and a rather small geometric albedo, $A=8 \%$. Note that such a low albedo is quite close to the average albedo of $\mathrm{C}$ type asteroids (e.g., Zellner and Gradie, 1976). Figure 2 shows that the NPB for scattering from independent grains (open symbols) is noticeably deeper than that for the deposited particles (filled symbols): $P_{\min } \approx-1.7 \%$ versus $P_{\min } \approx-0.5 \%$. Thus, the multiple scattering between particles forming a regolith substantially depolarizes the scattered radiation at small phase angles. It has to be stressed that this finding holds true for all the samples investigated in Shkuratov et al. (2004). However, in the case of bright samples, the depolarization effect is much higher; the decrease of amplitude of the NPB can be as much as an order of magnitude.

Interestingly, the conclusion on the depolarization effect 

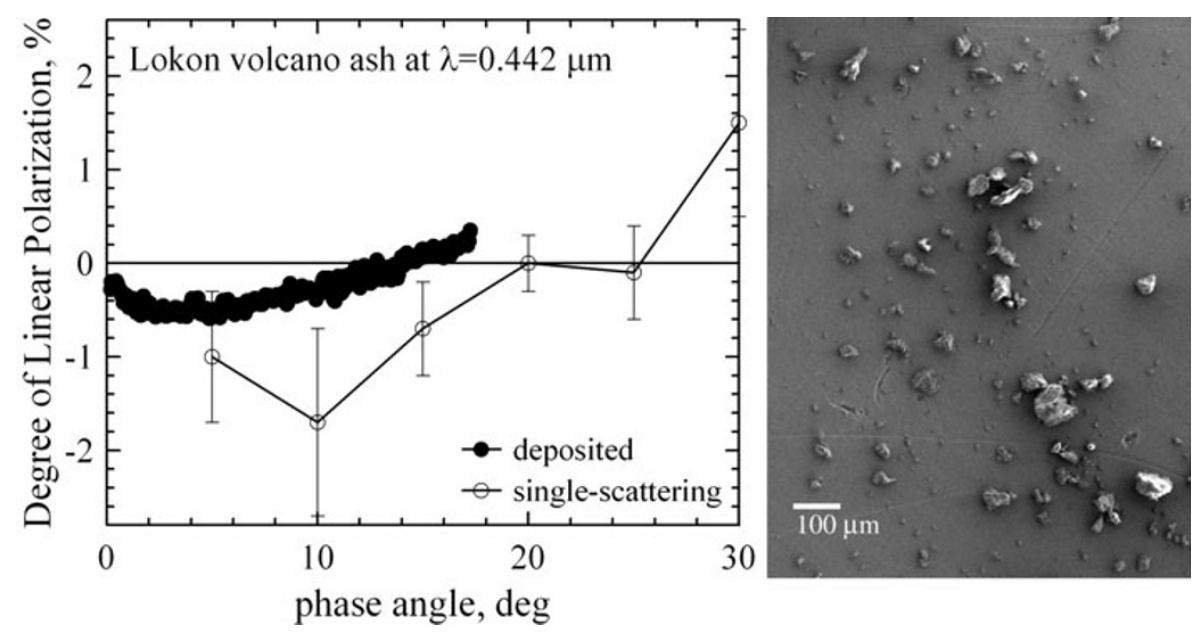

Fig. 2. Dependences of the degree of linear polarization on phase angle for a Lokon volcano ash sample. Open symbols are results for single-scattering particles; filled ones-for the regolith consisting of exactly the same particles. The SEM image on the right-hand panel shows the morphology of Lokon volcano ash particles. Measurement data are adapted from Shkuratov et al. (2004); whereas the SEM image is from Shkuratov et al. (2006).

of multiple scattering is consistent with the polarimetry of asteroids. For instance, E-type asteroids $(A \approx 50 \%)$ produce an NPB with $P_{\min } \approx-0.3 \%$; whereas, in the case of S-type asteroids $(A \approx 20 \%), P_{\min } \approx-0.7 \%$ (e.g., Belskaya et al., 2003). However, C-type asteroids $(A \approx 7 \%)$ produce the NPB with $P_{\min } \approx-1.8 \%$ (e.g., Zellner and Gradie, 1976). Notice also that, in general, the multiple scattering between particles in a regolith may produce the negative polarization at small phase angles. The comprehensive explanation for the origin of this effect was given independently by Shkuratov (1985) and Muinonen (1989). Sometimes, the effect is referred to as the polarization opposition effect (POE) in order to distinguish it from the NPB, though a different origin for the NPB and POE is not obvious (e.g., Shkuratov et al., 2002; Zubko et al., 2008). Nevertheless, in practice, the POE is attributed to those cases when the minimum of polarization is located at $\alpha \leq 3^{\circ}$ (e.g., Shkuratov et al., 2002). For instance, it is believed that the POE is responsible for the secondary minimum in phase curves of linear polarization observed at $\alpha=0.8-1.8^{\circ}$ for E-type asteroids (Rosenbush et al., 2009b).

As was shown in Fig. 2, there is a noticeable difference in the degree of linear polarization produced by the independently scattering particles and regoliths consisting of such particles. Therefore, the coincidence in the NPB of comets and C-type asteroids cannot be interpreted in terms of common properties of their dust particles. Moreover, the similarity in the polarization curves of comets and asteroids must testify to a large difference between cometary and asteroidal dust. On the other hand, this conclusion is inconsistent with the well-established similarity between IDPs originating in comets and asteroids (e.g., Brownlee, 1985); so, the resemblance in the negative polarization of comets and asteroids needs to be re-examined.

\section{The Resemblance of the NPBs of Comets and C-type Asteroids Revisited}

An obvious restriction of the aperture-averaged polarimetry of comets is that this completely ignores variations of the linear polarization through the coma. However, such variations have been known for sometime (e.g. Elvius, 1958). The variations of the linear polarization through a cometary coma were reported also by Martel (1960), Clarke (1971), Dollfus and Suchail (1987), Renard et al. (1996), Jockers et al. (1997), Tanga et al. (1997), Furusho et al. (1999), Hadamcik and Levasseur-Regourd (2003a), and many others. Note also that the variations of linear polarization in the inner coma (i.e., at distances from the nucleus of less than approximately $7000 \mathrm{~km}$ ) have been detected by the optical probe experiment (OPE) on board the Giotto space probe for comet 1P/Halley (Levasseur-Regourd et al., 1999) and 26P/Grigg-Skjellerup (McBride et al., 1997). The extended picture of the linear polarization variations in comae could be obtained from the imaging polarimetry of comets.

The most comprehensive imaging polarimetry of comets was reported by Hadamcik and Levasseur-Regourd (2003b), who present data for nine different comets. Their results have been confirmed by other observers. For instance, the bright comet C/1995 O1 (Hale-Bopp) attracted the attention of many observers across the world, and at least three other groups carried out imaging polarimetry of this comet, all giving similar results (i.e., Jockers et al., 1997; Tanga et al., 1997; Furusho et al., 1999). Also integrating imaging data over the whole coma compares well with aperture-averaged polarimetry (Hadamcik and Levasseur-Regourd, 2003b).

Polarimetric images of comets presented in Hadamcik and Levasseur-Regourd (2003b) reveal two distinctive features in the coma: a circumnucleus halo and jets or arcs (depending on the projection of coma upon the celestial sphere). The circumnucleus (or circumnuclear) halo is a relatively small area of the inner coma surrounding the nucleus; its radius typically does not exceed a few thousand kilometers. The circumnucleus halo produces a noticeable negative polarization with an amplitude of 6-7\%; whereas, the branch of negative polarization extends up to a phase angle $\alpha \approx 30^{\circ}$. Jets or arcs are outflows of highly-accelerated dust particles. For instance, in comet C/1995 O1 (HaleBopp), the projected velocity was estimated to be about $400 \mathrm{~m} / \mathrm{s}$ (Lisse et al., 1997). Unlike haloes, the degree of 

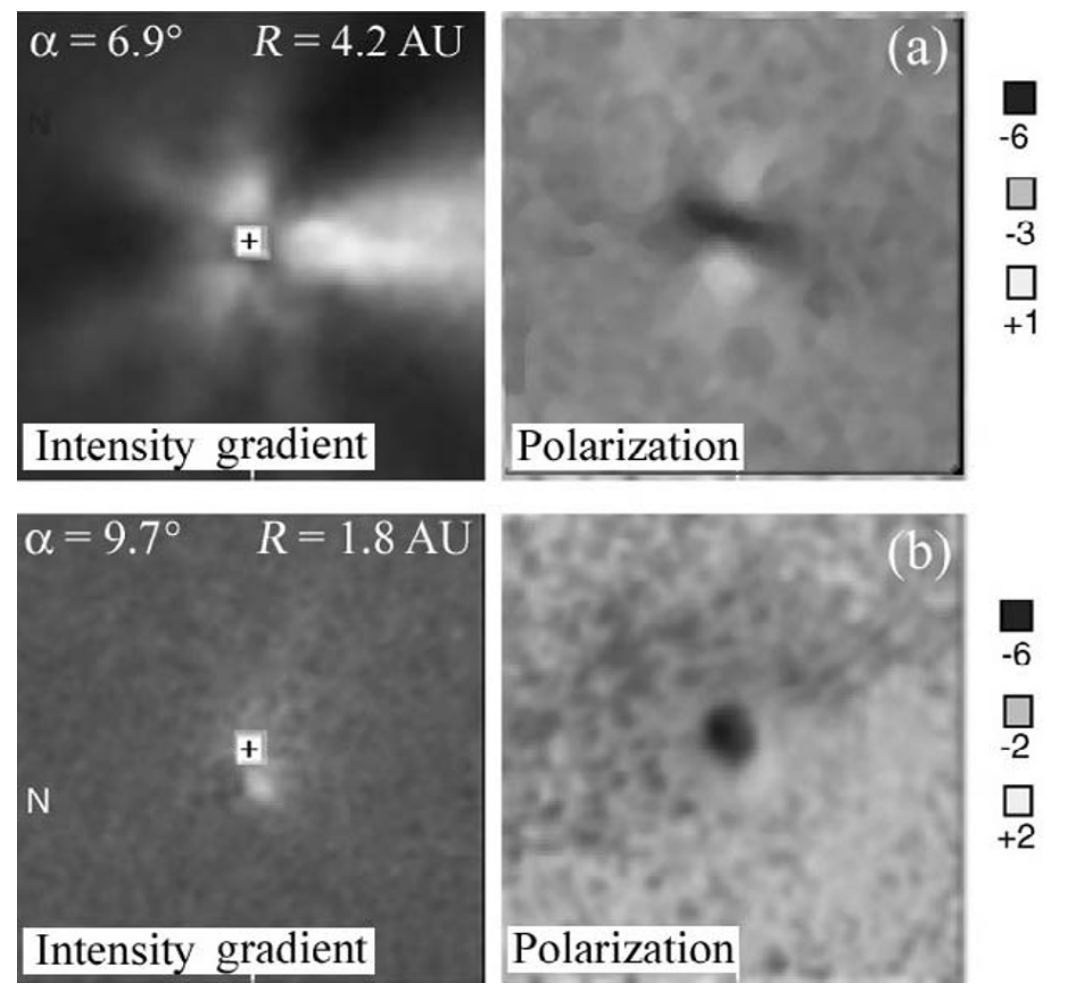

Fig. 3. Intensity gradient and polarimetric images of comets C/1995 O1 (Hale-Bopp) (top) and 81P/Wild (bottom) obtained at $\lambda=0.67 \mu \mathrm{m}$ (adapted from Hadamcik and Levasseur-Regourd (2003b)).

linear polarization in jets/arcs remains positive at all phase angles, including even small phase angles.

Figure 3 shows the intensity gradient (on the left) and polarimetric images for two different comets adapted from Hadamcik and Levasseur-Regourd (2003b). The upper row presents the data for comet C/1995 O1 (Hale-Bopp) and the bottom row for comet $81 \mathrm{P} /$ Wild. The images are 38000 and $9000 \mathrm{~km}$ for the upper and bottom frames, respectively. The phase angle $\alpha$ and heliocentric distance $R$ for the observation dates are given in the photometric images. In both polarimetric images, one can clearly see the circumnucleus haloes. Note also that the positive polarization, which is noticeably higher than the background, can be confidently associated with the jets apparent in photometric images. Nevertheless, one bright detail in the intensity gradient of comet C/1995 O1 (Hale-Bopp) is invisible in the polarimetric image.

Obviously, the average polarization in the whole coma is determined by contributions from halo, jets, and the rest of the coma. For instance, in the image corresponding to comet C/1995 O1 (Hale-Bopp), the highest values for positive and negative polarization are $(2 \pm 0.5) \%$ and $(-5 \pm 1) \%$, respectively; whereas, the polarization integrated over the whole coma is $(-0.4 \pm 0.4) \%$ (Hadamcik and Levasseur-Regourd, 2003b). Thus, in the apertureaveraged polarimetry of comets, a significant negative polarization in the circumnucleus halo could be disguised by high positive polarization in jets. It needs to be emphasized that a high jet-activity may completely hide a circumnuclear halo (Hadamcik and Levasseur-Regourd, 2003b). However, high jet-activity generally correlates with a small heliocentric distance of a comet; as a consequence, ground-based observation of haloes at large phase angles is a difficult problem.

It needs to be stressed that some reports on unusually high negative polarization in the very inner part of the coma can also be found for aperture-averaged polarimetric observations of comets. For example, Jockers and Kiselev (2002) found an unusually high negative polarization in comet C/2000 WM1 (LINEAR) at small phase angles. It is possible to outline two necessary conditions for apertureaveraged observations of circumnucleus haloes. The first implies that the circumnucleus halo is not hidden by jetactivity from the observer. However, weak jet-activity typically could be observed at a relatively large heliocentric distance $R$ of the comet. The second condition requires a small geocentric distance $\Delta$ of the comet. It increases the apparent brightness of the comet and, therefore, makes possible an observation with small-sized apertures. Moreover, a small geocentric distance increases spatial resolution.

Jockers and Kiselev (2002) measured the linear polarization in comet $\mathrm{C} / 2000 \mathrm{WM} 1$ (LINEAR) and found $P \approx$ $-2.4 \%$ at the phase angle $\alpha=13^{\circ}$. However, due to the small geocentric distance of the comet $\Delta=0.468$ A.U., the projected area in their measurements was only about 3750 $\times 3750 \mathrm{~km}$. Furthermore, during the observations, jets were not apparent in the coma (Jockers and Kiselev, 2002); to some extent, such a weak jet-activity could be explained by the non-small heliocentric distance $R=1.439$. The finding of unusually-high negative polarization was quite surprising for the authors and, unfortunately, they did not try to interpret it. Nevertheless, the finding in Jockers and Kiselev (2002) is consistent with the high negative polarization in circumnucleus haloes reviewed by Hadamcik and 


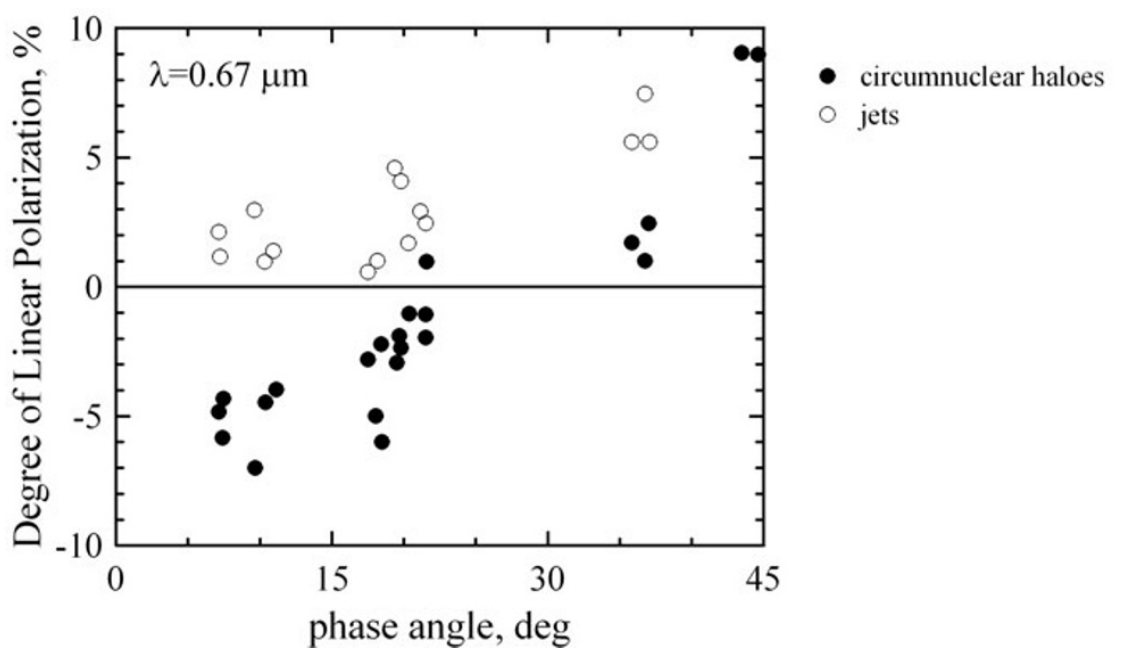

Fig. 4. Synthetic dependences of the degree of linear polarization on phase angle for the circumnucleus haloes (filled symbols) and jets (open symbols). Data are adapted from Hadamcik and Levasseur-Regourd (2003b).

Levasseur-Regourd (2003b).

Figure 4 presents synthetic dependences of the degree of linear polarization on phase angle for circumnucleus haloes and jets (data points adapted from Hadamcik and Levasseur-Regourd, 2003b). In Fig. 4, the minimum of polarization $P_{\min }$ and phase angle of polarization minimum $\alpha_{\text {min }}$ in the NPB of circumnucleus haloes can be estimated as approximately $-6 \%$ and $15^{\circ}$, respectively. Taking into account the depolarizing effect of multiple scattering in the regolith, which was discussed in Section 2, a significant difference of the NPBs in circumnucleus haloes (shown in Fig. 4) and C-type asteroids (in Fig. 1) could indicate some similarity in the properties of their dust. On the other hand, the absence of the NPB in cometary jets suggests a substantial difference in the properties of dust forming the jets and regolith in C-type asteroids.

\section{Interpretation of Imaging Polarimetry of Comets}

Unfortunately, there has been little effort to date to interpret the imaging polarimetry of comets. For instance, Hadamcik and Levasseur-Regourd (2003b) attributed the high negative polarization in circumnucleus haloes to "more compact particles with different ices." The first quantitative analysis of synthetic phase curves of the linear polarization measured in circumnucleus haloes was done by Zubko et al. (2009). There, the impact of absorption on light scattering, by irregularly-shaped particles with sizes comparable with wavelength, were studied. While the real part of the refractive index $m$ was fixed, $\operatorname{Re}(m)=1.5$, the imaginary part was varied from 0 to 1.3 . It was found that an increase of material absorption quickly reduces the amplitude of the negative polarization, so the effect disappears at $\operatorname{Im}(m)>0.1$; whereas, high negative polarization with $P_{\min }=-6 \%$ implies an absence of highly-absorbing materials with $\operatorname{Im}(m) \leq 0.02$ in circumnucleus haloes. However, in Zubko et al. (2009), only single-sized particles were considered; whereas, a more realistic simulation must include a size distribution of dust particles and this is adopted in this section for the analysis of the NPB in circumnucleus haloes.

As in Zubko et al. (2009), we use the so-called agglomerated debris particles model for the shape of cometary dust particles. The generation algorithm for these model particles has been previously described (e.g. in Zubko et al., 2006, 2009). On average, agglomerated debris particles are equi-dimensional with a rather-low packing density of material $\rho=0.236$. Constituent grains, as well as being overall agglomerates, have a truly irregular morphology. Note that the agglomerate structure and irregular morphology are widely thought to be features of cometary dust. Example images of agglomerated debris particles are shown in Fig. 5. As can be seen, some constituent grains do not necessarily touch other parts of the agglomerated debris particles, though they are located very close to each other. However, as was shown in Zubko et al. (2008), in the case of irregularly-shaped particles, the absence of the direct contact between constituent grains does not affect significantly the light-scattering properties; this is especially the case for the NPB. More images of agglomerated debris particles can be found in Zubko et al. (2006, 2009).

Light scattering by agglomerated debris particles is simulated with the discrete dipole approximation (DDA, e.g., Draine and Flatau, 1994; Zubko et al., 2010; and references therein). We use our own programming realization of the DDA, which reveals quite a good accuracy of computation (Penttilä et al., 2007). In this paper, we present results for three refractive indices $m=1.6+0.0005 i, 1.6+0.02 i$, and $1.6+0.1 i$; differing only in the imaginary part.

Light scattering by particles comparable with wavelength is determined by the ratio of particle radius $r$ to the wavelength $\lambda$ of the incident radiation, which is referred to as the size parameter (e.g., Bohren and Huffman, 1983). The size parameter is denoted by $x$ and expressed as: $x=2 \pi r / \lambda$. However, in the case of irregularly-shaped particles, the meaning of radius needs to be specified. We attribute the radius $r$ to a sphere circumscribing a target particle. Note that, if necessary, the size parameter $x$ referring to the circumscribing sphere can be easily transformed to the size parameter of equal-volume sphere $x_{\text {eq }}$ through the packing 

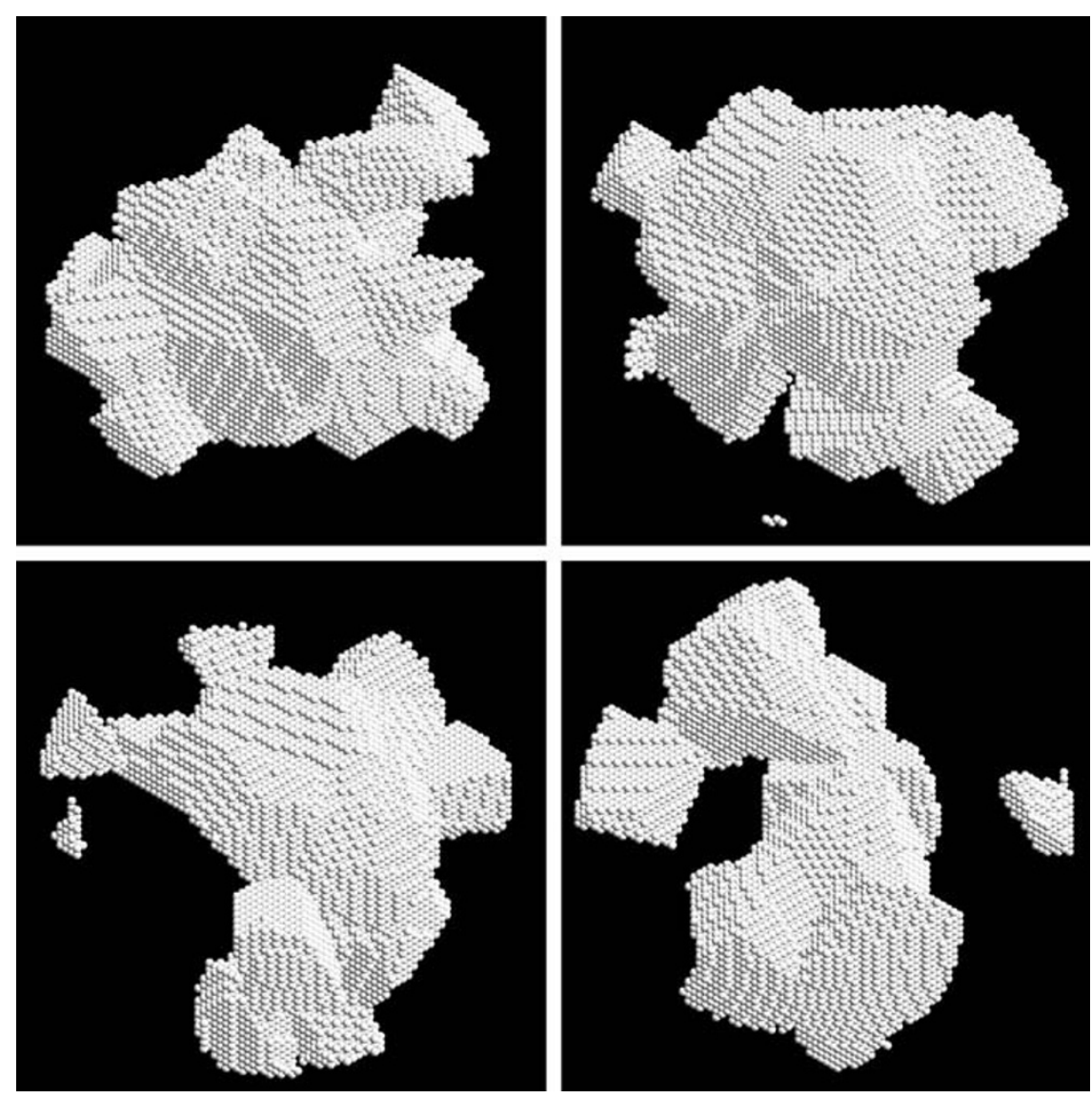

Fig. 5. Images of four samples of agglomerated debris particles.

density $\rho$ as follows: $x_{\mathrm{eq}}=\rho^{1 / 3} x$. Through this study, the size parameter $x$ is varied from 1 to 26 (in the case of $m=1.6+0.0005 i), 28(m=1.6+0.02 i)$, and 32 $(m=1.6+0.1 i)$. The difference in the upper range limit of $x$ is caused by limitations in the DDA convergence. At wavelength $\lambda=0.67 \mu \mathrm{m}$, the range of $x$ corresponds to particle radii from $0.1 \mu \mathrm{m}$ to $2.8,3$, and $3.4 \mu \mathrm{m}$, respectively.

Light-scattering properties of agglomerated debris particles are averaged over sample shapes and orientations at each set of $m$ and $x$; using a minimum of 500 sample particle shapes. Light scattering by each sample particle has been computed for one random orientation of the incident electromagnetic wave and averaged over 100 scattering planes evenly distributed around the propagation direction of the incident light. We continue averaging over particle shape, while fluctuations of the standard deviation of the degree of linear polarization over the entire range of phase angle $\alpha$ exceed $1 \%$; therefore, the actual number of sample particles considered very often exceeds 500. More details on the averaging over scattering planes and control of the averaging quality can be found in Zubko et al. (2008).

Light-scattering properties of agglomerated debris particles are also averaged over particle size with power-law size distribution $r^{-a}$; whereas, the power index $a$ is varied from 1 to 4 with a step of 0.1 . Note that the studied range of $a$ is consistent with the results of in situ measurement of comet 1P/Halley with VeGa-1 and 2 spacecrafts (Mazets et $a l ., 1986)$. For instance, as was found in the case of comet
1P/Halley, the power index $a$ varied from 1.5 to 3.4 over the mass range of cometary dust particles from $10^{-19}$ to larger than $10^{-12} \mathrm{~kg}$. Interestingly, the lower range limit of $a$ corresponds to the most lightweight particles.

Figure 6 presents the results of numerical simulation of light scattering by agglomerated debris particles. The left panel shows the minimum of the negative polarization $P_{\min }$ and the right panel-the phase angle of the minimum of polarization $\alpha_{\min }$; both are functions of the power index $a$. Open symbols correspond to weakly-absorbing particles with $\operatorname{Im}(m)=0.0005$ and 0.02 ; whereas, the filled onesto highly-absorbing particles with $\operatorname{Im}(m)=0.1$. The dashed line in each panel shows the corresponding characteristic for the circumnucleus haloes. As can be seen in the left panel, only weakly-absorbing particles may produce the necessary amplitude of the NPB. Moreover, this occurs only in the range of $a$ from 1 to 2 . Thus, the present extended analysis confirms the previous findings in Zubko et al. (2009) for single-sized particles at $\operatorname{Re}(m)=1.5$. Particles with refractive indices $\operatorname{Re}(m)=1.5-1.6$ and $\operatorname{Im}(m)=$ 0-0.02 can be confidently attributed to Mg-rich silicates (e.g., Dorschner et al., 1995). On the other hand, Mg-rich silicates were indeed detected within the in situ study of comet 1P/Halley (e.g., Jessberger, 1999) and the laboratory investigation of dust samples returned by the Stardust mission from the coma of comet 81P/Wild (e.g., Hörz et al., 2006). However, it is impossible to immediately rule out the presence in circumnucleus haloes of specific organic materials, such as, for instance, kerogen (Khare et al., 1990) or 

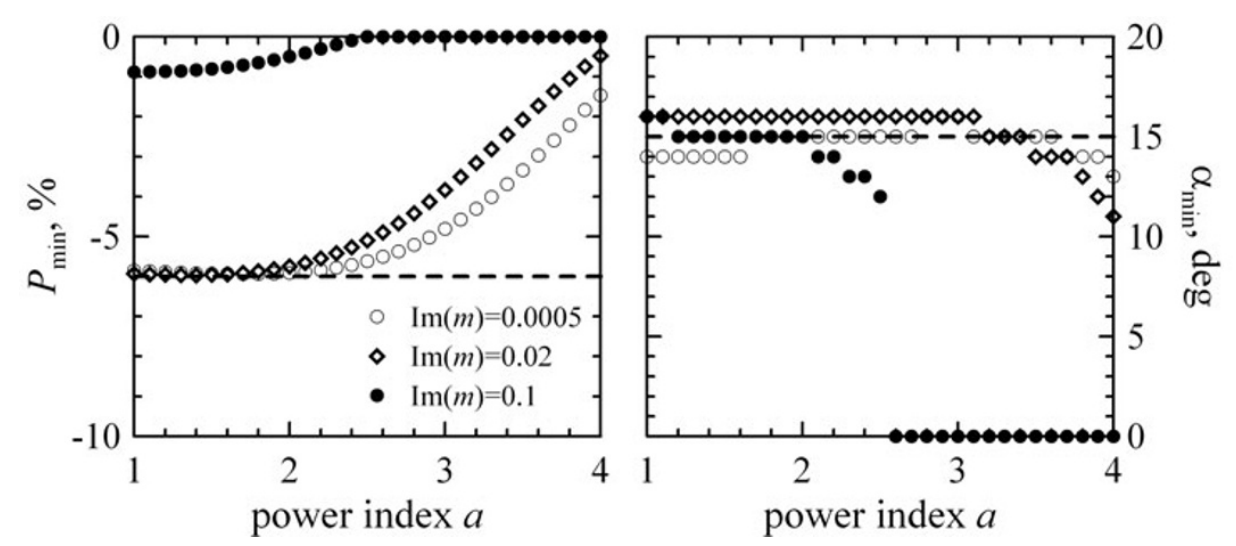

Fig. 6. The minimum of the negative polarization $P_{\min }$ and phase angle of the minimum of the negative polarization $\alpha_{\min }$ versus the index $a$ in power-law size distribution of agglomerated debris particles. Open symbols correspond to weakly-absorbing materials with $m=1.6+0.0005 i$ and $1.6+0.02 i$; whereas, filled symbols present the case of high absorption $m=1.6+0.1 i$.

ice tholin (Khare et al., 1993). Nevertheless, one can conclude that the organic material based on that of the Greenberg model (e.g., Jenniskens, 1993) cannot exist in considerable quantities in the circumnucleus haloes; neither can hydrogenated carbon (e.g., Duley, 1984).

As one can see in Fig. 6, weakly-absorbing agglomerated debris particles fit the amplitude of the NPB in the circumnucleus haloes in the power index range $a=1-2$. Under the assumption of material density $\rho_{\text {mat }}=3 \mathrm{~g} / \mathrm{cm}^{3}$, the mass of agglomerated debris particles studied in Fig. 6 could be estimated between $10^{-17} \mathrm{~kg}$ and $10^{-14} \mathrm{~kg}$. However, in this range of mass, in situ measurements reveal the index in power-law size distribution to be $a=1.5-2.5$ (Mazets et al., 1986). Thus, the range of $a$ that provides a good fit of $P_{\min }$ in the circumnucleus haloes and, simultaneously, is consistent with in situ studies of cometary dust, is $a=1.5-$ 2. It needs to be emphasized that, in the given range of $a$, the computed $\alpha_{\min }$ also fits quite well with that observed in the circumnucleus haloes. Thus, both principal characteristics of the NPB in our simulation are consistent with the observations. It is interesting to note that our estimation of the power index $a$ for dust particles in circumnucleus haloes significantly differs from the indirect estimations of average power index for the whole coma (e.g., Jockers, 1997). On the other hand, the dust in circumnucleus haloes is not the most abundant constituent of the coma.

Finally, we can consider the question of the density of dust particles in circumnucleus haloes. As previously noted, Hadamcik and Levasseur-Regourd (2003b) assumed a compact structure of dust particles in the haloes. While agglomerated debris particles fit the principal parameters characterizing the NPB, their material density is not too high. Indeed, assuming the material density of the solid silicates to be $3.5 \mathrm{~g} / \mathrm{cm}^{3}$ and the packing density of material to be 0.236 , the material density in agglomerated debris particles is about $0.83 \mathrm{~g} / \mathrm{cm}^{3}$. However, according to the laboratory study of craters in aluminum foil of the Stardust collector, the material density of cometary dust is between 0.3 to $3 \mathrm{~g} / \mathrm{cm}^{3}$ (Hörz et al., 2006). Therefore, agglomerated debris particles have to be classified as being fluffy particles rather than compact.

\section{Summary}

The discussion presented in this paper can be summarized as follows. Though the aperture-averaged linear polarization of comets does nearly coincide with that of Ctype asteroids at small phase angles, it is seen as a random coincidence. The similarity cannot be considered as evidence for common properties of dust particles in comets and asteroids. According to laboratory measurements, light scattering by independent dust particles is substantially different from that by regoliths consisting of exactly the same particles. In particular, the negative polarization of independent particles is at least a few times higher than that of the regolith.

Aperture-averaged linear polarization in comets cannot be attributed to a single type of dust particle. There are at least two types of cometary dust particles with substantially different polarimetric properties: dust particles forming cometary jets and those forming the circumnucleus halo. Dust particles in jets produce only positive polarization through all phase angles; whereas, those in the halo reveal a significant negative polarization branch at phase angles $\alpha \leq 30^{\circ}$. In the latter case, the amplitude of the NPB can be as high as $6 \%$. However, this value is more than three times the amplitude of the NPB in C-type asteroids $\left(\left|P_{\min }\right| \approx 1.8 \%\right)$. Taking into account findings in the experimental study of light scattering by independent and deposited particles, one can assume common properties of dust particles in the cometary circumnucleus haloes and regoliths of C-type asteroids.

Interpretation of the high negative polarization in circumnucleus haloes leads to a number of important conclusions on the properties of dust particles in those haloes. First of all, the dust particles consist of weakly-absorbing material. The real part of the refractive index $\operatorname{Re}(m)$ is $1.5-1.6$, and its imaginary part has to be limited to $\operatorname{Im}(m) \leq 0.02$. Such a refractive index is well consistent with $\mathrm{Mg}$-rich silicates, but not with highly-absorbing organic materials nor amorphous carbon. The index in power-law size distribution of dust particle has to be squeezed to the range $a=1.5-2$. This estimation agrees with findings of in situ measurements of dust in the comet $1 \mathrm{P} /$ Halley, but it substantially differs from estimations of the index $a$ for whole comae. 
Acknowledgments. This research was partially supported by the Academy of Finland (contract 127461) and by the NASA program for Outer Planets Research (grant NNX10AP93G). I am grateful to my colleagues Dr. G. Videen, Prof. T. Yamamoto, Dr. H. Kimura, Prof. K. Muinonen, Prof. Yu. G. Shkuratov, and Dr. E. Hadamcik for numerous discussions of various aspects of this work. A special thanks to Dr. A. Ovcharenko for the digitized polarimetric data of the regolith consisting of Lokon volcano ash particles. Also, I am thankful to Prof. J. H. Hough for valuable comments on this work and his kind help with the correction of my English.

\section{References}

Belskaya, I. N., V. G. Shevchenko, N. N. Kiselev, Yu. N. Krugly, N. M. Shakhovskoy, Yu. S. Efimov, N. M. Gaftonyuk, A. Cellino, and R. Gil-Hutton, Opposition polarimetry and photometry of S- and E-type asteroids, Icarus, 166, 276-284, 2003.

Bohren, C. F. and D. R. Huffman, Absorption and Scattering of Light by Small Particles, 530 pp., Wiley, New York, 1983.

Brownlee, D. E., Cosmic dust-collection and research, Ann. Rev. Earth Planet. Sci., 13, 147-173, 1985.

Chernova, G. P., N. N. Kiselev, and K. Jockers, Polarimetric characteristics of dust particles as observed in 13 comets-Comparisons with asteroids, Icarus, 103, 144-158, 1993.

Clarke, D., Polarization measurements of the head of comet Beunett (1969i), Astron. Astrophys., 14, 90-94, 1971.

Dollfus, A. and J.-L. Suchail, Polarimetry of grains in the coma of P/Halley. I-Observations, Astron. Astrophys., 187, 669-688, 1987.

Dorschner, J., B. Begemann, T. Henning, C. Jaeger, and H. Mutschke, Steps toward interstellar silicate mineralogy. II. Study of $\mathrm{Mg}$-Fe-silicate glasses of variable composition, Astron. Astrophys., 300, 503-520, 1995.

Draine, B. T. and P. J. Flatau, The discrete dipole approximation for scattering calculations, J. Opt. Soc. Am. A, 11, 1491-1499, 1994.

Duley, W. W., Refractive indices for amorphous carbon, Astrophys. J., 287, 694-696, 1984.

Elvius, A., Preliminary results of polarization measurements in comets, Arkiv för Astronomii, 2, 195-197, 1958

Furusho, R., B. Suzuki, N. Yamamoto, H. Kawakita, T. Sasaki, Y. Shimizu, and T. Kurakami, Imaging polarimetry and color of the inner coma of comet Hale-Bopp (C/1995 O1), Publ. Astron. Soc. Jpn., 51, 363-373, 1999.

Ganesh, S., U. C. Joshi, K. S. Baliyan, and M. R. Deshpande, Polarimetric observations of the comet Hale-Bopp, Astron. Astrophys. Suppl. Ser., 129, 489-493, 1998.

Gustafson, B. A. S. and L. Kolokolova, A systematic study of light scattering by aggregate particles using the microwave analog technique: Angular and wavelength dependence of intensity and polarization, J. Geophys. Res., 104, 31711-31720, 1999.

Hadamcik, E. and A. C. Levasseur-Regourd, Dust evolution of comet C/1995 O1 (Hale-Bopp) by imaging polarimetric observations, Astron. Astrophys., 403, 757-768, 2003a.

Hadamcik, E. and A. C. Levasseur-Regourd, Imaging polarimetry of cometary dust: different comets and phase angles, J. Quant. Spectr. Rad. Trans., 79-80, 661-678, 2003b.

Hadamcik, E., J.-B. Renard, A. C. Levasseur-Regourd, J. Lasue, G. Alcouffe, and M. Francis, Light scattering by agglomerates: Interconnecting size and absorption effects (PROGRA ${ }^{2}$ experiment), J. Quant. Spectr. Rad. Trans., 110, 1755-1770, 2009.

Hörz, F., R. Bastien, J. Borg, J. P. Bradley, J. C. Bridges, D. E. Brownlee, M. J. Burchell, M. Chi, M. J. Cintala, Z. R. Dai, Z. Djouadi, G. Dominguez, and 32 colleagues, Impact features on Stardust: Implications for comet 81P/Wild 2 dust, Science, 314, 1716-1719, 2006.

Jenniskens, P., Optical constants of organic refractory residue, Astron. Astrophys., 274, 653-661, 1993.

Jessberger, E. K., Rocky cometary particulates: their elemental isotopic and mineralogical ingredients, Space Sci. Rev., 90, 91-97, 1999.

Jockers, K., Observations of scattered light from cometary dust and their interpretation, Earth Moon Planets, 79, 221-245, 1997.

Jockers, K. and N. Kiselev, Wavelength dependence of polarization of comet $\mathrm{C} / 2000 \mathrm{WM} 1$ (LINEAR) in the negative and positive polarization branches, in Proceedings of Asteroids, Comets, Meteors-ACM 2002, edited by B. Warmbein, 567-570, (Berlin, Germany, 29 July-2 August, 2002), 2002

Jockers, K., V. K. Rosenbush, T. Bonev, and T. Credner, Images of polarization and colour in the inner coma of comet Hale-Bopp, Earth Moon
Planets, 78, 373-379, 1997.

Joshi, U. C., K. S. Baliyan, and S. Ganesh, Polarization studies of comet C/2000 WM1 (LINEAR), Astron. Astrophys., 405, 1129-1135, 2003.

Joshi, U. C., S. Ganesh, and K. S. Baliyan, Optical polarimetry and photometry of comet 17P/Holmes, Mon. Not. R. Astron. Soc., 402, 2744 2752, 2010.

Khare, B. N., W. R. Thompson, C. Sagan, E. T. Arakawa, C. Meisse, and I. Gilmour, Optical constants of kerogen from 0.15 to 40 (m: comparison with meteoritic organics, Proc. Lunar Planet. Sci. Conf., 21, 627-628, 1990

Khare, B. N., W. R. Thompson, L. Cheng, C. Chyba, C. Sagan, E. T. Arakawa, C. Meisse, and P. S. Tuminello, Production and optical constants of ice tholin from charged particle irradiation of $(1: 6) \mathrm{C}_{2} \mathrm{H}_{6} / \mathrm{H}_{2} \mathrm{O}$ at $77 \mathrm{~K}$, Icarus, 103, 290-300, 1993.

Kikuchi, S., Y. Mikami, T. Mukai, S. Mukai, and J. H. Hough, Polarimetry of comet P/Halley, Astron. Astrophys., 187, 689-692, 1987.

Kimura, H., Light-scattering properties of fractal aggregates: numerica calculations by a superposition technique and the discrete-dipole approximation, J. Quant. Spectr. Rad. Trans., 70, 581-594, 2001.

Kimura, H., L. Kolokolova, and I. Mann, Optical properties of cometary dust. Constraints from numerical studies on light scattering by aggregate particles, Astron. Astrophys., 407, L5-L8, 2003.

Kiselev, N. N. and G. P. Chernova, Phase functions of polarization and brightness and the nature of cometary atmosphere particles, Icarus, $\mathbf{4 8}$, 473-481, 1981.

Lasue, J. and A. C. Levasseur-Regourd, Porous irregular aggregates of sub-micron sized grains to reproduce cometary dust light scattering observations, J. Quant. Spectr. Rad. Trans., 100, 220-236, 2006.

Levasseur-Regourd, A. C., E. Hadamcik, and J. B. Renard, Evidence for two classes of comets from their polarimetric properties at large phase angles, Astron. Astrophys., 313, 327-333, 1996.

Levasseur-Regourd, A. C., N. McBride, E. Hadamcik, and M. Fulle, Similarities between in situ measurements of local dust light scattering and dust flux impact data within the coma of 1P/Halley, Astron. Astrophys., 348, 636-641, 1999.

Lindqvist, H., K. Muinonen, and T. Nousiainen, Light scattering by coated Gaussian and aggregate particles, J. Quant. Spectr. Rad. Trans., 110, 1398-1410, 2009

Lisse, C. M., Y. R. Fernández, M. F. A’Hearn, T. Kostiuk, T. A. Livengood, H. U. Käufl, W. F. Hoffmann, A. Dayal, M. E. Ressler, M. S. Hanner, G. G. Fazio, J. L. Hora, S. B. Peschke, E. Grün, and L. K. Deutsch, Infrared observations of dust emission from comet Hale-Bopp, Earth Moon Planets, 78, 251-257, 1997

Lumme, K. and J. Rahola, Light scattering by porous dust particles in the discrete-dipole approximation, Astrophys. J., 425, 653-667, 1994

Martel, M. T., Variations de la polarisation observée du 18 au 29 août dans la comète 1957 d pour des régions situées à moins de 4' du noyau, Annales d'Astrophysique, 23, 480-497, 1960.

Mazets, E. P., R. L. Aptekar, S. V. Golenetskii, Yu. A. Guryan, A. V. Dyachkov, V. N. Ilyinskii, V. N. Panov, G. G. Petrov, A. V. Savvin, R. Z. Sagdeev, I. A. Sokolov, N. G. Khavenson, V. D. Shapiro, and V. I. Shevchenko, Comet Halley dust environment from SP-2 detector measurements, Nature, 321, 276-278, 1986.

McBride, N., S. F. Green, A. C. Levasseur-Regourd, B. Goidet-Devel, and J.-B. Renard, The inner dust coma of comet 26P/Grigg-Skjellerup: multiple jets and nucleus fragments?, Mon. Not. R. Astron. Soc., 289 535-553, 1997.

Muinonen, K., Electromagnetic scattering by two interacting dipoles, in Proc. 1989 URSI Electromagnetic Theory Sympos., 428-430, Stockholm, Sweden, 1989.

Muinonen, K., E. Zubko, J. Tyynelä, Yu. G. Shkuratov, and G. Videen, Light scattering by Gaussian random particles with discrete-dipole approximation, J. Quant. Spectr. Rad. Trans., 106, 360-377, 2007.

Muñoz, O., H. Volten, J. F. de Haan, W. Vassen, and J. W. Hovenier, Experimental determination of scattering matrices of olivine and Allende meteorite particles, Astron. Astrophys., 360, 777-788, 2000.

Muñoz, O., H. Volten, J. W. Hovenier, M. Min, Yu. G. Shkuratov, J. P. Jalava, W. J. van der Zande, and L. B. F. M. Waters, Experimental and computational study of light scattering by irregular particles with extreme refractive indices: hematite and rutile, Astron. Astrophys., 446, 525-535, 2006

Nousiainen, T., Optical modeling of mineral dust particles: A review, $J$ Quant. Spectr. Rad. Trans., 110, 1261-1279, 2009.

Penttilä, A., E. Zubko, K. Lumme, K. Muinonen, M. A. Yurkin, B. Draine, J. Rahola, A. G. Hoekstra, and Yu. Shkuratov, Comparison between discrete dipole implementations and exact techniques, J. Quant. Spectr. 
Rad. Trans., 106, 417-436, 2007.

Petrova, E. V., K. Jockers, and N. N. Kiselev, A negative branch of polarization for comets and atmosphereless celestial bodies and the light scattering by aggregate particles, Sol. Syst. Res., 35, 390-399, 2000.

Renard, J.-B., E. Hadamcik, and A. C. Levasseur-Regourd, Polarimetric CCD imaging of comet 47P/Ashbrook-Jackson and variability of polarization in the inner coma of comets, Astron. Astrophys., 316, 263-269, 1996.

Renard, J.-B., M. Francis, E. Hadamcik, D. Daugeron, B. Couté, B. Gaubicher, and M. Jeannot, Scattering properties of sands. 2. Results for sands from different origins, Appl. Opt., 49, 3552-3559, 2010.

Rosenbush, V. K., A. E. Rosenbush, and M. S. Dement'ev, Comets Okazaki-Levy-Rundenko (1989 XIX) and Levy (1990 XX): Polarimetry and stellar occultations, Icarus, 108, 81-91, 1994.

Rosenbush, V., N. Kiselev, L. Kolokolova, S. Velichko, F. Velichko, K. Antoniuk, and S. Kolesnikov, Polarization properties of odd comet 17P/Holmes, J. Quant. Spectr. Rad. Trans., 110, 1719-1725, 2009 a.

Rosenbush, V. K., V. G. Shevchenko, N. N. Kiselev, A. V. Sergeev, N. M. Shakhovskoy, F. P. Velichko, S. V. Kolesnikov, and N. V. Karpov, Polarization and brightness opposition effects for the E-type Asteroid 44 Nysa, Icarus, 201, 655-665, 2009 b.

Shkuratov, Yu. G., On the origin of the opposition effect and negative polarization for cosmic bodies with solid surface, Astronomicheskii Circular, 1400, 3-6, 1985 (in Russian).

Shkuratov, Yu., A. Ovcharenko, E. Zubko, O. Miloslavskaya, K. Muinonen, J. Piironen, R. Nelson, W. Smythe, V. Rosenbush, and P. Helfenstein, The opposition effect and negative polarization of structural analogs for planetary regoliths, Icarus, 159, 396-416, 2002.

Shkuratov, Yu., A. Ovcharenko, E. Zubko, H. Volten, O. Munoz, and G. Videen, The negative polarization of light scattered from particulate surfaces and of independently scattering particles, J. Quant. Spectr. Rad. Trans., 88, 267-284, 2004.

Shkuratov, Yu., S. Bondarenko, A. Ovcharenko, C. Pieters, T. Hiroi, H. Volten, O. Munoz, and G. Videen, Comparative studies of the reflectance and degree of linear polarization of particulate surfaces and independently scattering particles, J. Quant. Spectr. Rad. Trans., 100, 340-358, 2006.

Tanga, P., A. Cellino, and M. Di Martino, Imaging polarimetry of comet Hale-Bopp (C/1995 O1) around perihelion, Earth Moon Planets, 78, 359-364, 1997.

Vilaplana, R., F. Moreno, and A. Molina, Study of the sensitivity of sizeaveraged scattering matrix elements of nonspherical particles to changes in shape, porosity and refractive index, J. Quant. Spectr. Rad. Trans., 100, 415-428, 2006.

Volten, H., O. Muñoz, E. Rol, J. F. de Haan, W. Vassen, J. W. Hovenier, K. Muinonen, and T. Nousiainen, Scattering matrices of mineral aerosol particles at $441.6 \mathrm{~nm}$ and $632.8 \mathrm{~nm}$, J. Geophys. Res., 106, 1737517402, 2001.

Yanamandra-Fisher, P. A. and M. S. Hanner, Optical properties of nonspherical particles of size comparable to the wavelength of light: Application to comet dust, Icarus, 138, 107-128, 1999.

Zellner, B. and J. Gradie, Minor planets and related objects. XXPolarimetric evidence for the albedos and compositions of 94 asteroids, Astron. J., 81, 262-280, 1976.

Zubko, E., Yu. Shkuratov, M. Hart, J. Eversole, and G. Videen, Backscattering and negative polarization of agglomerate particles, Opt. Lett., 28, 1504-1506, 2003.

Zubko, E., Yu. Shkuratov, M. Hart, J. Eversole, and G. Videen, Backscattering of agglomerate particles, J. Quant. Spectr. Rad. Trans., 88, 163172, 2004.

Zubko, E., D. Petrov, Yu. Shkuratov, and G. Videen, Discrete dipole approximation simulations of scattering by particles with hierarchical structure, Appl. Opt., 44, 6479-6485, 2005.

Zubko, E., Yu. Shkuratov, N. Kiselev, and G. Videen, DDA simulations of light scattering by small irregular particles with various structure, $J$. Quant. Spectr. Rad. Trans., 101, 416-434, 2006.

Zubko, E., K. Muinonen, Yu. G. Shkuratov, G. Videen, and T. Nousiainen, Scattering of light by roughened Gaussian random particles, J. Quant. Spectr. Rad. Trans., 106, 604-615, 2007.

Zubko, E., Yu. Shkuratov, M. Mishchenko, and G. Videen, Light scattering in a finite multi-particle system, J. Quant. Spectr. Rad. Trans., 109, 2195-2206, 2008

Zubko, E., H. Kimura, Yu. Shkuratov, K. Muinonen, T. Yamamoto, H. Okamoto, and G. Videen, Effect of absorption on light scattering by agglomerated debris particles, J. Quant. Spectr. Rad. Trans., 110, 17411749, 2009.

Zubko, E., D. Petrov, Ye. Grynko, Yu. Shkuratov, H. Okamoto, K. Muinonen, T. Nousiainen, H. Kimura, T. Yamamoto, and G. Videen, Validity criteria of the discrete dipole approximation, Appl. Opt., 49, 1267-1279, 2010.

E. Zubko (e-mail: ezubko@rambler.ru) 